\title{
On Two Alternative Axiomatizations of Lattices by McKenzie and Sholander
}

\author{
Adam Grabowski \\ Institute of Informatics \\ University of Białystok \\ Poland
}

\author{
Damian Sawicki \\ Institute of Informatics \\ University of Białystok \\ Poland
}

\begin{abstract}
Summary. The main result of the article is to prove formally that two sets of axioms, proposed by McKenzie and Sholander, axiomatize lattices and distributive lattices, respectively. In our Mizar article we used proof objects generated by Prover9. We continue the work started in 17, 21, and 13] of developing lattice theory as initialized in [22] as a formal counterpart of [1]. Complete formal proofs can be found in the Mizar source code of this article available in the Mizar Mathematical Library (MML).
\end{abstract}

MSC: 03B35 68T99 06B05 06D05

Keywords: lattice; distributive lattice; lattice axioms

MML identifier: ROBBINS5, version: 8.1.08 5.52.1328

\section{INTRODUCTION}

For years, automated theorem provers have proven to be useful tool to solve quite complex problems dealing with axiomatizations of various systems appearing in mathematics. Let us recall here the Robbins problem about the alternative axiomatization of Boolean algebras: this was probably the first time lots of mathematicians have heard of EQP/OTTER [15. The Mizar system, via interface ot2miz [19] allows for the automated translation of OTTER (or Prover9) proof objects to allows such proofs to be included into the Mizar repository. Among the examples of such areas of mathematics within the Mizar Mathematical Library (MML) [1] explored by means of Prover9 we can give 
either the aforementioned solution of the Robbins problem [7] according to [3], various short systems for ortholattices [21] inspired by [14], or axiom systems for Boolean algebras in terms of the Sheffer stroke [13. An overview of the mechanization of lattice theory in MML can be found in [5]. The initial idea of this development was to provide a formal counterpart of [11] (or, more recently, [12]) or [2] and this Mizar challenge is alive for over thirty years now [9]. This is also quite feasible taking into account automatic treatment of the equality predicate in Mizar [10, and the equational axiomatics for lattices is strongly preferred in the MML over that based on the ordering relation [4, although we created a common - fully formal - Mizar framework where both can be used in parallel [8].

In 1951, in his paper [20] Marlow Sholander showed that the necessary and sufficient condition for an algebra $\langle L, \sqcup, \sqcap\rangle$ to be a distributive lattice is to satisfy one of the following sets of axioms:

$$
\begin{gathered}
a=a \sqcup(a \sqcap b), \\
a \sqcup(b \sqcap c)=(c \sqcup a) \sqcap(b \sqcup a) ;
\end{gathered}
$$

or, dually

$$
\begin{gathered}
a=a \sqcap(a \sqcup b), \\
a \sqcap(b \sqcup c)=(c \sqcap a) \sqcup(b \sqcap a)
\end{gathered}
$$

for arbitrary elements $a, b, c$ of $L$.

We call the latter formula the Sholander axiom, and show in the first section, that together with the other one, which corresponds with the Mizar adjective join-absorbing, it implies all remaining standard axioms for distributive lattices as defined in [22]. The theorem stating full equivalence of both axiom sets is under number (11) in the present article.

Ralph McKenzie's [17] axiomatization of lattices consists of four formulas:

$$
\begin{gathered}
x \sqcup(y \sqcap(x \sqcap z))=x \\
x \sqcap(y \sqcup(x \sqcup z))=x \\
((y \sqcap x) \sqcup(x \sqcap z)) \sqcup x=x \\
((y \sqcup x) \sqcap(x \sqcup z)) \sqcap x=x
\end{gathered}
$$

where $x, y, z$ are arbitrary elements of the carrier of $\langle L, \sqcup, \sqcap\rangle$. These formulas were introduced in Section 2 in definitions (Def. 2) - (Def. 5), respectively, and the full equivalence of these four axioms with the classical axiomatics from [22] is proven as theorem (15) providing also appropriate registration of clusters allowing for automated reuse of both sets. Such approach is useful especially in 
the areas which use lattice theory as a kind of metalanguage, e.g., rough sets [6].

Our work can be seen as a step towards a Mizar support for [16] or [18], where original proof objects by OTTER/Prover9 were used.

\section{Sholander Axiom for Distributive Lattices}

From now on $L$ denotes a non empty lattice structure and $v_{4}, v_{5}, v_{6}, v_{7}, w_{3}$, $v, w_{2}, w_{1}, w_{0}, z, y, x$ denote elements of $L$.

Let us consider $L$. We say that $L$ satisfies Sholander axiom if and only if

(Def. 1) for every $x, y$, and $z, x \sqcap(y \sqcup z)=(z \sqcap x) \sqcup(y \sqcap x)$.

Let us consider $x$. Now we state the propositions:

(1) If $L$ is join-absorbing and for every $x, z$, and $y, x \sqcap(y \sqcup z)=(z \sqcap x) \sqcup(y \sqcap x)$, then $x \sqcap x=x$.

(2) If $L$ is join-absorbing and for every $x, z$, and $y, x \sqcap(y \sqcup z)=(z \sqcap x) \sqcup(y \sqcap x)$, then $x \sqcup x=x$. The theorem is a consequence of (1).

Let us consider $x$ and $y$. Now we state the propositions:

(3) If $L$ is join-absorbing and for every $x, z$, and $y, x \sqcap(y \sqcup z)=(z \sqcap x) \sqcup(y \sqcap x)$, then $x \sqcap y=y \sqcap x$. The theorem is a consequence of (2).

(4) If $L$ is join-absorbing and for every $x, z$, and $y, x \sqcap(y \sqcup z)=(z \sqcap x) \sqcup(y \sqcap x)$, then $x \sqcup y=y \sqcup x$. The theorem is a consequence of (1).

(5) Suppose $L$ is join-absorbing and for every $x, z$, and $y, x \sqcap(y \sqcup z)=$ $(z \sqcap x) \sqcup(y \sqcap x) .(x \sqcap y) \sqcap z=x \sqcap(y \sqcap z)$. The theorem is a consequence of $(1),(2),(4)$, and (3).

(6) If for every $y$ and $x, x \sqcap(x \sqcup y)=x$, then for every $x$ and $y, x \sqcap(x \sqcup y)=x$.

(7) Suppose $L$ is join-absorbing and for every $x, z$, and $y, x \sqcap(y \sqcup z)=$ $(z \sqcap x) \sqcup(y \sqcap x) . x \sqcup(x \sqcap y)=x$. The theorem is a consequence of (1), (3), and (4).

Let us consider $x, y$, and $z$. Now we state the propositions:

(8) Suppose $L$ is join-absorbing and for every $x, z$, and $y, x \sqcap(y \sqcup z)=$ $(z \sqcap x) \sqcup(y \sqcap x)$. Then $(x \sqcup y) \sqcup z=x \sqcup(y \sqcup z)$. The theorem is a consequence of (1), (3), (7), (2), (5), and (4).

(9) Suppose $L$ is join-absorbing and for every $x$, and $y, x \sqcap(y \sqcup z)=$ $(z \sqcap x) \sqcup(y \sqcap x)$. Then $x \sqcap(y \sqcup z)=(x \sqcap y) \sqcup(x \sqcap z)$. The theorem is a consequence of (4) and (3).

(10) Suppose $L$ is join-absorbing and for every $x, z$, and $y, x \sqcap(y \sqcup z)=$ $(z \sqcap x) \sqcup(y \sqcap x)$. Then $x \sqcup(y \sqcap z)=(x \sqcup y) \sqcap(x \sqcup z)$. The theorem is a consequence of (5), (1), (4), (8), (2), and (3). 
From now on $L$ denotes a distributive, join-commutative, meet-commutative, non empty lattice structure and $x, y, z$ denote elements of $L$.

Now we state the propositions:

(11) $x \sqcap(y \sqcup z)=(z \sqcap x) \sqcup(y \sqcap x)$.

(12) Let us consider a non empty lattice structure $L$. Then $L$ is a distributive lattice if and only if $L$ is join-absorbing and satisfies Sholander axiom. The theorem is a consequence of (11), (9), (3), (4), (5), (8), and (7).

Let us observe that every non empty lattice structure which is join-absorbing and satisfies Sholander axiom is also distributive and lattice-like and every non empty lattice structure which is distributive, join-commutative, and meetcommutative satisfies also Sholander axiom.

\section{Four Axioms for Lattices Proposed by McKenzie}

From now on $L$ denotes a non empty lattice structure and $w_{3}, v, w_{2}, w_{1}, w_{0}$, $z, y, x$ denote elements of $L$.

Let us consider $L$. We say that $L$ satisfies first McKenzie axiom if and only if

(Def. 2) for every $y, z$, and $x, x \sqcup(y \sqcap(x \sqcap z))=x$.

We say that $L$ satisfies second McKenzie axiom if and only if

(Def. 3) for every $y, z$, and $x, x \sqcap(y \sqcup(x \sqcup z))=x$.

We say that $L$ satisfies third McKenzie axiom if and only if

(Def. 4) for every $z, y$, and $x,((x \sqcap y) \sqcup(y \sqcap z)) \sqcup y=y$.

We say that $L$ satisfies fourth McKenzie axiom if and only if

(Def. 5) for every $z, y$, and $x,((x \sqcup y) \sqcap(y \sqcup z)) \sqcap y=y$.

Now we state the propositions:

(13) Suppose $L$ satisfies first McKenzie axiom and second McKenzie axiom and for every $z, y$, and $x,((x \sqcap y) \sqcup(y \sqcap z)) \sqcup y=y$ and for every $z, y$, and $x,((x \sqcup y) \sqcap(y \sqcup z)) \sqcap y=y$. Then

(i) for every $y$ and $x, x \sqcap(x \sqcup y)=x$, and

(ii) for every $y$ and $x, x \sqcup(x \sqcap y)=x$, and

(iii) $L$ is join-commutative, meet-commutative, meet-associative, and joinassociative.

(14) Suppose $L$ is join-commutative, join-associative, meet-commutative, and meet-associative and for every $y$ and $x, x \sqcap(x \sqcup y)=x$ and for every $y$ and $x, x \sqcup(x \sqcap y)=x$. Then

(i) for every $y$, $z$, and $x, x \sqcup(y \sqcap(x \sqcap z))=x$, and 
(ii) for every $y, z$, and $x, x \sqcap(y \sqcup(x \sqcup z))=x$, and

(iii) for every $z, y$, and $x,((x \sqcap y) \sqcup(y \sqcap z)) \sqcup y=y$, and

(iv) for every $z, y$, and $x,((x \sqcup y) \sqcap(y \sqcup z)) \sqcap y=y$.

Let $L$ be a non empty lattice structure. We say that $L$ satisfies four McKenzie axioms if and only if

(Def. 6) $L$ satisfies first McKenzie axiom, second McKenzie axiom, third McKenzie axiom, and fourth McKenzie axiom.

One can verify that every non empty lattice structure which satisfies four McKenzie axioms satisfies also first McKenzie axiom, second McKenzie axiom, third McKenzie axiom, and fourth McKenzie axiom and every non empty lattice structure which satisfies first McKenzie axiom, second McKenzie axiom, third McKenzie axiom, and fourth McKenzie axiom satisfies also four McKenzie axioms.

From now on $L$ denotes a non empty lattice structure.

Now we state the proposition:

(15) $L$ is a lattice if and only if $L$ satisfies four McKenzie axioms. The theorem is a consequence of (14) and (13).

Let us observe that every non empty lattice structure which is lattice-like satisfies also four McKenzie axioms and every non empty lattice structure which satisfies four McKenzie axioms is also lattice-like.

\section{REFERENCES}

[1] Grzegorz Bancerek, Czesław Byliński, Adam Grabowski, Artur Korniłowicz, Roman Matuszewski, Adam Naumowicz, and Karol Pąk. The role of the Mizar Mathematical Library for interactive proof development in Mizar. Journal of Automated Reasoning, 61(1):9-32, 2018. do1:10.1007/s10817-017-9440-6

[2] Garrett Birkhoff. Lattice Theory. Providence, Rhode Island, New York, 1967.

[3] B. I. Dahn. Robbins algebras are Boolean: A revision of McCune's computer-generated solution of Robbins problem. Journal of Algebra, 208:526-532, 1998.

[4] B.A. Davey and H.A. Priestley. Introduction to Lattices and Order. Cambridge University Press, 2002.

[5] Adam Grabowski. Mechanizing complemented lattices within Mizar system. Journal of Automated Reasoning, 55:211-221, 2015. doi:10.1007/s10817-015-9333-5.

[6] Adam Grabowski. Lattice theory for rough sets - a case study with Mizar. Fundamenta Informaticae, 147(2-3):223-240, 2016. doi 10.3233/FI-2016-1406

[7] Adam Grabowski. Robbins algebras vs. Boolean algebras Formalized Mathematics, 9(4): 681-690, 2001.

[8] Adam Grabowski and Markus Moschner. Managing heterogeneous theories within a mathematical knowledge repository. In Andrea Asperti, Grzegorz Bancerek, and Andrzej Trybulec, editors, Mathematical Knowledge Management Proceedings, volume 3119 of Lecture Notes in Computer Science, pages 116-129. Springer, 2004. doi:10.1007/978-3540-27818-4_9. 3rd International Conference on Mathematical Knowledge Management, Bialowieza, Poland, Sep. 19-21, 2004.

[9] Adam Grabowski, Artur Korniłowicz, and Adam Naumowicz. Four decades of Mizar. Journal of Automated Reasoning, 55(3):191-198, 2015. doi:10.1007/s10817-015-9345-1 
[10] Adam Grabowski, Artur Korniłowicz, and Christoph Schwarzweller. Equality in computer proof-assistants. In Ganzha, Maria and Maciaszek, Leszek and Paprzycki, Marcin, editor, Proceedings of the 2015 Federated Conference on Computer Science and Information Systems, volume 5 of ACSIS-Annals of Computer Science and Information Systems, pages 45-54. IEEE, 2015. doi $10.15439 / 2015$ F229.

[11] George Grätzer. General Lattice Theory. Academic Press, New York, 1978.

[12] George Grätzer. Lattice Theory: Foundation. Birkhäuser, 2011.

[13] Violetta Kozarkiewicz and Adam Grabowski. Axiomatization of Boolean algebras based on Sheffer stroke Formalized Mathematics, 12(3):355-361, 2004.

[14] W. McCune, R. Padmanabhan, M. A. Rose, and R. Veroff. Automated discovery of single axioms for ortholattices. Algebra Universalis, 52(4):541-549, 2005.

[15] William McCune. Prover9 and Mace4 2005-2010.

[16] William McCune and Ranganathan Padmanabhan. Automated Deduction in Equational Logic and Cubic Curves. Springer-Verlag, Berlin, 1996.

[17] Ralph McKenzie. Equational bases for lattice theories. Mathematica Scandinavica, 27: 24-38, 1970. doi $10.7146 /$ math.scand.a-10984.

[18] Ranganathan Padmanabhan and Sergiu Rudeanu. Axioms for Lattices and Boolean Algebras. World Scientific Publishers, 2008.

[19] Piotr Rudnicki and Josef Urban. Escape to ATP for Mizar. In First International Workshop on Proof eXchange for Theorem Proving-PxTP 2011, 2011.

[20] Marlow Sholander. Postulates for distributive lattices. Canadian Journal of Mathematics, 3:28-30, 1951. doi 10.4153/CJM-1951-003-5.

[21] Wioletta Truszkowska and Adam Grabowski. On the two short axiomatizations of ortholattices. Formalized Mathematics, 11(3):335-340, 2003.

[22] Stanisław Żukowski. Introduction to lattice theory Formalized Mathematics, 1(1):215222, 1990 